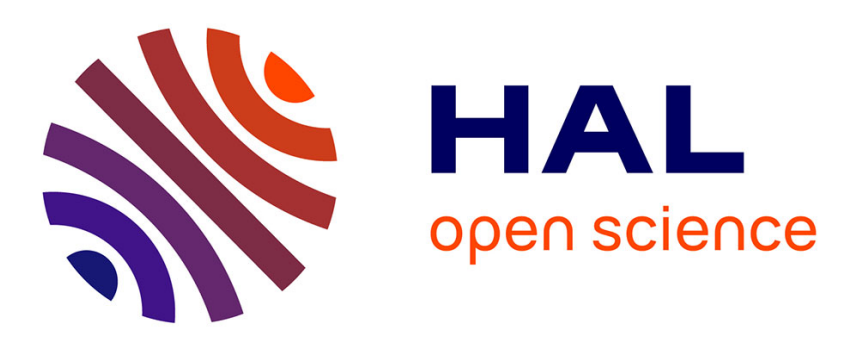

\title{
Postural dependence of human locomotion during gait initiation
}

\author{
Marie-Laure Mille, Martin Simoneau, Mark W. Rogers
}

\section{To cite this version:}

Marie-Laure Mille, Martin Simoneau, Mark W. Rogers. Postural dependence of human locomotion during gait initiation. Journal of Neurophysiology, 2014, 112 (12), pp.3095-3103. 10.1152/jn.00436.2014 . hal-01438448

\section{HAL Id: hal-01438448 \\ https://hal.science/hal-01438448}

Submitted on 23 May 2018

HAL is a multi-disciplinary open access archive for the deposit and dissemination of scientific research documents, whether they are published or not. The documents may come from teaching and research institutions in France or abroad, or from public or private research centers.
L'archive ouverte pluridisciplinaire HAL, est destinée au dépôt et à la diffusion de documents scientifiques de niveau recherche, publiés ou non, émanant des établissements d'enseignement et de recherche français ou étrangers, des laboratoires publics ou privés. 


\title{
Postural dependence of human locomotion during gait initiation
}

\author{
Marie-Laure Mille, ${ }^{1,2,3}$ Martin Simoneau, ${ }^{4}$ and Mark W. Rogers ${ }^{5}$ \\ ${ }^{1}$ Université de Toulon, La Garde, France; ${ }^{2}$ Aix Marseille Université, Centre National de la Recherche Scientifique, Institut \\ des Sciences du Mouvement Unité Mixte de Recherche 7287, Marseille, France; ${ }^{3}$ Department of Physical Therapy \\ and Human Movement Sciences, Northwestern University Medical School, Chicago, Illinois; ${ }^{4}$ Department of Social \\ and Preventive Medicine, Laval University Medical School, Québec, Canada; and ${ }^{5}$ Department of Physical \\ Therapy and Rehabilitation Science, University of Maryland School of Medicine, Baltimore, Maryland
}

Submitted 11 June 2014; accepted in final form 12 September 2014

Mille ML, Simoneau M, Rogers MW. Postural dependence of human locomotion during gait initiation. J Neurophysiol 112: 3095-3103, 2014. First published September 17, 2014; doi:10.1152/jn.00436.2014.-The initiation of human walking involves postural motor actions for body orientation and balance stabilization that must be effectively integrated with locomotion to allow safe and efficient transport. Our ability to coordinately adapt these functions to environmental or bodily changes through error-based motor learning is essential to effective performance. Predictive compensations for postural perturbations through anticipatory postural adjustments (APAs) that stabilize mediolateral (ML) standing balance normally precede and accompany stepping. The temporal sequencing between these events may involve neural processes that suppress stepping until the expected stability conditions are achieved. If so, then an unexpected perturbation that disrupts the ML APAs should delay locomotion. This study investigated how the central nervous system (CNS) adapts posture and locomotion to perturbations of ML standing balance. Healthy human adults initiated locomotion while a resistance force was applied at the pelvis to perturb posture. In experiment 1, using random perturbations, step onset timing was delayed relative to the APA onset indicating that locomotion was withheld until expected stability conditions occurred. Furthermore, stepping parameters were adapted with the APAs indicating that motor prediction of the consequences of the postural changes likely modified the step motor command. In experiment 2, repetitive postural perturbations induced sustained locomotor aftereffects in some parameters (i.e., step height), immediate but rapidly readapted aftereffects in others, or had no aftereffects. These results indicated both rapid but transient reactive adaptations in the posture and gait assembly and more durable practice-dependent changes suggesting feedforward adaptation of locomotion in response to the prevailing postural conditions.

balance; coordination; locomotion; posture; motor adaptation

THE PROVERBIAL EXPRESSION "the first step is always the hardest" undoubtedly applies as much to the literal task of human locomotion as it does to life's many other challenges. Although bipedal terrestrial locomotion is a seemingly simple routine activity, it requires motor adaptation and learning throughout the life span together with the integration of posture and locomotion. For example, human standing necessitates that the postural orientation of the body segments is controlled in concert with stable equilibrium. The complexities of remaining upright during locomotion in the changing natural environment therefore require that these control functions be effectively integrated and adapted together.

Address for reprint requests and other correspondence: M. W. Rogers, Dept. of Physical Therapy \& Rehabilitation Science, School of Medicine, Univ. of Maryland, 100 Penn St., Allied Health Bldg. 205D, Baltimore, MD 21201 (e-mail: mrogers@som.umaryland.edu).
The integration of posture and locomotion is particularly evident during the transition between standing still and walking (Brunt et al. 1991). Herein, centrally organized anticipatory postural adjustments (APAs) involving a sequence of muscle activations and changes in the ground reaction forces serve to overcome the resting inertia of the body and propel the body center of mass (CoM) forward and laterally for weight transfer and mediolateral (ML) stability before stepping (Brenière et al. 1987; Rogers and Pai 1990, 1993). Thus the ML APAs minimize in advance the tendency for the body to fall laterally and downwards at first step liftoff (Rogers et al. 2001).

Two perspectives have been posited to account for the coordination between posture and intended actions including locomotion (Massion 1992; Schepens and Drew 2003). First, APAs and the initiation of stepping could involve two independent but parallel motor commands that engage both elements sequentially, with a fixed inherent delay in triggering locomotion. In this "independent model" (Fig. 1, left), an "unexpected" perturbation opposing the ML APA would not affect the onset timing release of stepping and temporal sequencing would remain constant. Alternatively, this coordination could be organized interactively as demonstrated for occulomotor and manual movements (Scarchilli and Vercher 1999). In this "interactive model" (Fig. 1, right), the neuronal circuits for stepping are suppressed until the APAs have achieved an anticipated stable state that, once satisfied, would release the step. Applying unexpected perturbations should delay locomotion because the expected postural conditions are achieved later than anticipated. The first aim of this study tested these two hypotheses by randomly perturbing the MLAPA before stepping.

A related issue involves how posture and locomotion coupling is adapted to postural constraints during repeated perturbations that disrupt their normal coordination. If the temporal sequencing between APA onset and stepping is disrupted for a sufficiently long period of time with repetitive perturbations, then adaptation should occur. With adaptation, "aftereffects" would appear immediately after the withdrawal of the perturbation where the modified temporal sequencing would need to be "deadapted" gradually through practicing unperturbed stepping to regain the original behavioral relationship (Bastian 2008). The second aim tested the hypothesis that if the initiation of walking depends on the prevailing postural state conditions (i.e., interactive model), then repeated perturbations that alter APA characteristics should durably change the onset timing release and possibly other parameters of locomotion. Alternatively, the 
INDEPENDENT Model

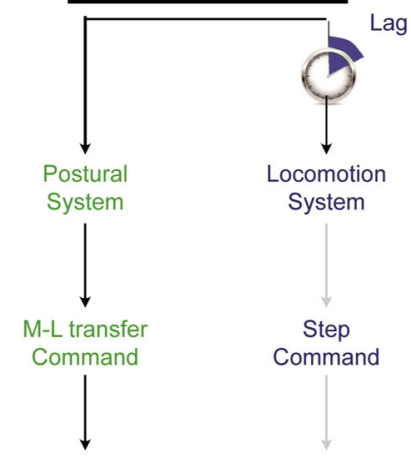

Lateral Hip EMG Leg EMG

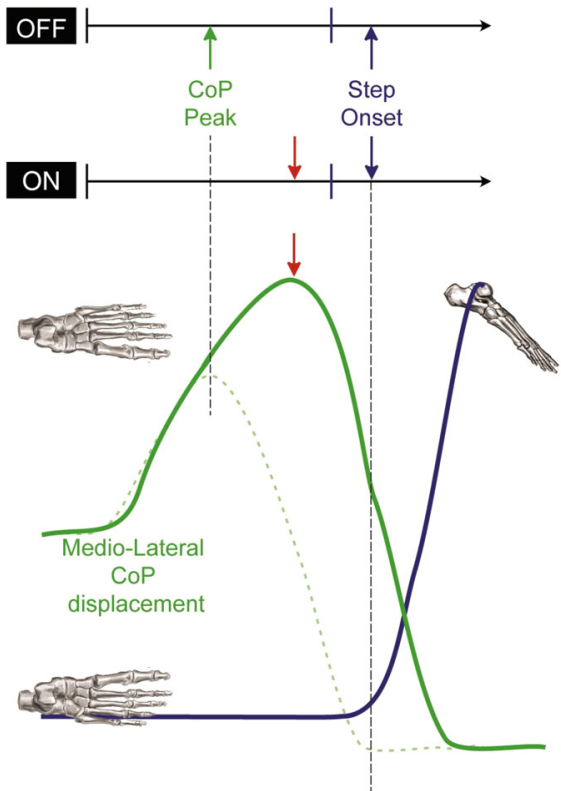

INTERACTIVE Model

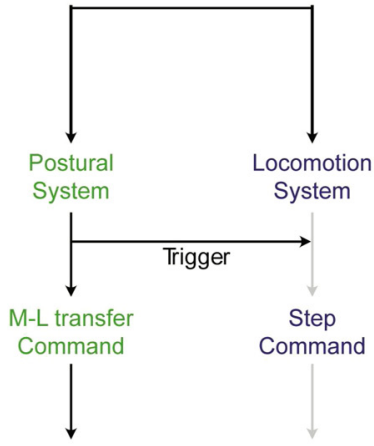

Lateral Hip EMG Leg EMG

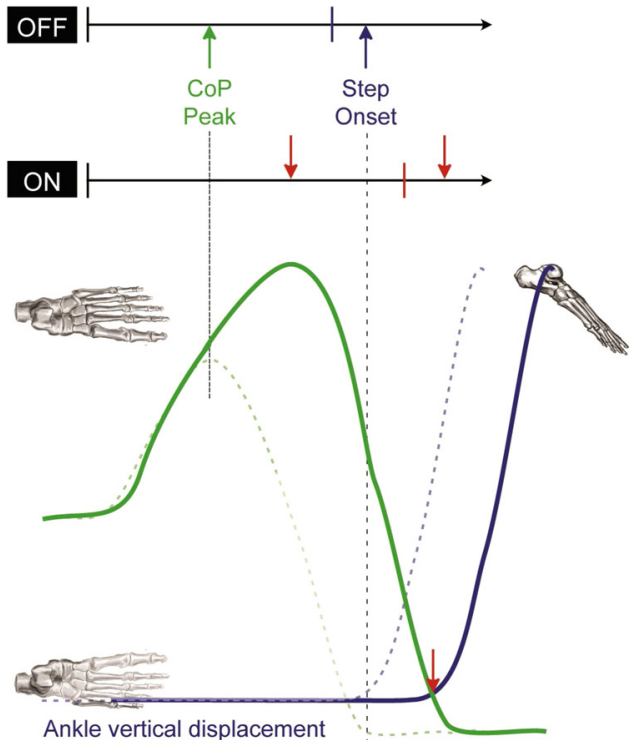

Fig. 1. Models for the posture and locomotion coupling. Two hypotheses for the sequencing of posture and locomotion during the initiation of walking tested by perturbing the postural weight transfer before stepping. OFF represents the normal unperturbed time course of the postural (green) and locomotor (blue) events occurring at the initiation of walking, and $\mathrm{ON}$ indicates the predicted time course of the same events when a passive frictional resistance is applied to the pelvis in the frontal plane to delay the time of occurrence of the mediolateral (ML) center of pressure (CoP) peak (red arrows). According to a 1st hypothesis (left), the postural and locomotor events could be sequenced by sending 2 independent motor commands to engage both systems with a fixed inherent delay (lag) in the release of locomotion to allow the ML weight transfer to occur before the onset of stepping. In this independent model of coordination, an unexpected perturbation of the postural system would not be taken into account by the locomotion system, such that the step command (gray bars) and step onset (gray arrows) would not be modified. Alternatively (right), these events could be sequenced in an interactive way such that locomotion onset might be triggered when anticipated postural state conditions have been reached. In this case, the same unexpected postural perturbation should delay the step command and onset since expected postural prerequisites would not be achieved. Bottom: schematic illustration of the postural ML CoP displacement (green) and the step ankle vertical displacement (blue). The usual normal profile observed during unperturbed step initiation is indicated by the dashed lines, and the solid lines represent the expected changes of these events according to both hypotheses.

absence of aftereffects would indicate that posture and locomotion coupling is mediated online through feedbackbased mechanisms. Preliminary results have been presented in abstract form (Mille et al. 2002).

\section{MATERIALS AND METHODS}

\section{Subjects}

Eleven healthy women $21-29$ yr old (mean age $23.3 \mathrm{yr} \pm 2.5$ ) with the right dominant leg participated in this study. All participants were naive about the purpose of the experiment and gave informed consent for a protocol approved by the Institutional Review Board of Northwestern University. None of them reported neurological, muscular, or sensory impairment.

\section{Experimental Protocol}

Each subject was instructed to initiate stepping as fast as possible beginning with their right leg in response to a light signal and to walk rapidly for three or four steps. The subjects walked on a path where foot traces of their normal gait pattern were marked before beginning the experiments to provide a guideline for maintaining a consistent gait pattern over repeated trials. Before each experiment, subjects were instructed to try to maintain the same gait velocity regardless of what might happen. To avoid premature anticipation of the "go" cue to begin stepping, catch trials (i.e., trials without the light cue) were inserted between normal trials but were not recorded. Subjects consistently followed the instructions, and none of them initiated stepping during these catch trials. A belt attached at the level of the subject's pelvis, and connected by a cable to an electromagnetic brake torque motor (Warner Electric; model MPB70-1), was used to perturb the 
ML weight transfer. The motor was engaged at the same time as the light cue and was stopped when the subject stepped (i.e., when the vertical force under the stepping foot was equal to zero). The perturbation corresponded to a lateral frictional force of $\sim 10 \%$ of the subject's body weight. No sound cues due to activation of the motor were transmitted to the subjects. All subjects participated in two experimental sessions performed on separate days.

Experiment 1. In the first session, subjects were not aware that their ML weight shift could be resisted momentarily or that the perturbation would be introduced randomly in $25 \%$ of the 60 trials. Subjects were regularly reminded to react as quickly as possible to the light cue and to walk fast, regardless of what might happen. Each subject completed 10 baseline trials (BASE) as a control condition, 45 trials with the brake off (OFF condition), and 15 trials where the brake unexpectedly resisted their ML weight shift (ON condition).

Experiment 2. The second session was performed between 3-7 days after the first one. The same instructions used for experiment 1 were given to the subjects and were regularly repeated. Following 10 preadaptation (PRE) baseline trials without the perturbation, subjects underwent 100 "adaptation" trials with the brake systematically on. Adaptation trials were organized by blocks of 10 trials. The perturbation was then unexpectedly removed for 10 postadaptation trials (POST).

\section{Data Collection}

The subjects stood on two separate force platforms (Advanced Mechanical Technology, Newton, MA) that recorded the ground reaction forces under each foot at a sampling of $500 \mathrm{~Hz}$. Ground forces were used to calculate net center of pressure $(\mathrm{CoP})$ in both the ML and the anteroposterior (AP) directions. Positions of 17 passive markers were recorded using a six-camera infrared motion analysis system (Motus Peak Performance System, Englewood, CO) sampling at $60 \mathrm{~Hz}$. Markers were placed symmetrically on the top of the toe, between the second and third metatarsal; the lateral malleolus; the knee joint line posterior to the lateral femoral condyle; the greater trochanter; the acromion; the lateral epicondyle of the elbow; the dorsum of wrist in the middle distance between both styloid processes; the ear canal; and one was placed on the crown of the head. The three-dimensional kinematics of 12 body segments was used to estimate the body CoM position and motion. Electromyographic (EMG) activity of six muscles of the stepping leg (i.e., the dominant leg) was recorded using surface electrodes with onsite preamplifiers $($ gain $\times 35$ ): the tibialis anterior, gastronemius medialis, soleus, rectus femoris, tensor fasciae latae, and the gluteus medius. Signals from the bipolar active electrodes were band-pass filtered $(20-250 \mathrm{~Hz})$ and amplified before digital recording at $500 \mathrm{~Hz}$. Kinetics, kinematics, and EMG data were recorded for $5 \mathrm{~s}$.

\section{Data Analysis}

Customized event detection algorithms and interactive graphical analysis programs (MatLab 6.0; MathWorks, Natick, MA) were used to analyze the data (Mille et al. 2005). Onset, amplitude, and duration of the APAs were derived from the net ML CoP displacement. The onset was defined as the beginning of the CoP displacement from baseline in the ML direction based on the first derivative (i.e., when the first derivative becomes continuously greater than zero). The amplitude was computed as the maximum step side displacement (i.e., lateral displacement peak under the stepping side) from the baseline and the duration as the elapsed time from the onset to the peak amplitude. The onset and the maximal lateral displacement of the CoM towards the stance leg were also analyzed to quantify the efficiency of the APA.

The onset and end of the step were identified from the vertical velocity of the right ankle marker. Step length and step width were assessed by determining the displacement of the ankle between step onset and end in the AP and ML directions, respectively. First step clearance was calculated as the maximum vertical displacement of this marker.

Onset of EMG activity relative to the onset of the APAs was identified automatically, using the algorithm proposed by Lidierth (1986) during locomotion but could be adjusted manually when necessary. This algorithm relies on a threshold voltage comparison followed by an analysis of the EMG burst duration. A burst of EMG activity was identified when the rectified EMG was higher than its mean value during baseline (first $500 \mathrm{~ms}$ of recording) plus 8 SD. When this occurred, the algorithm identified this point as an onset only if the burst was at least $70 \mathrm{~ms}$ in duration and not longer than $1,000 \mathrm{~ms}$, with transient decreases below the threshold not longer than $10 \mathrm{~ms}$. These parameters were chosen as they appeared well suited for this type of movement studies (van Boxtel et al. 1993).

\section{Statistical Analyses}

A nonparametric Friedman ANOVA was used to test the effects of the perturbation. When a significant main effect of the perturbation was found, post hoc comparisons were made using the two-tailed Wilcoxon signed rank test for a paired sample with Bonferroni correction. In experiment 1, comparisons were made between the three conditions. In experiment 2, the preadaptation trials (PRE) were compared with the first block of 10 adaptation trials (A1) and with the postadaptation trials (POST), whereas the last block of 10 adaptation trials (A10) was compared with the first adaptation block (A1) and with postadaptation trials (POST). To assess possible aftereffects due to the removal of the perturbation, we compared the preadaptation trials to the first postadaptation trial (T1) and each consecutive blocks of three trials (T2-4; T5-7; T8-10) using a one-tailed Wilcoxon signed rank. An alpha level of 0.05 after Bonferroni correction was considered statistically significant.

\section{RESULTS}

When subjects were cued to initiate rapid stepping, APAs invariably involved an initial lateral displacement of the net CoP towards the step limb side that was associated with phasic activation of the lateral hip muscles (i.e., gluteus medius or tensor fascia latae) to propel the CoM towards the single stance side (Fig. 2). Concomitantly, in the AP direction, an initial posterior displacement of the net CoP was associated with activation of tibialis anterior that propelled the CoM forward to begin walking (Mann et al. 1979; Breniere et al. 1987; Crenna and Frigo 1991). These APAs normally depend on the initial postural weight bearing conditions related to the CoM position (Mille and Mouchnino 1998). However, no differences were observed in the initial position of the CoM in either the ML direction [Friedman $\mathrm{Chi}^{2}(n=11, \mathrm{dl}=2)=1.28 ; P<0.53$ ] or the AP direction [Friedman $\mathrm{Chi}^{2}{ }_{(n=11, \mathrm{dl}=2)}=2.37 ; P<0.31$ ]. On average, the CoM was centered near the midline between the two feet (means \pm SD; $0.70 \pm 0.71 \mathrm{~cm}$ ) and slightly in front of the ankles $(6.25 \pm 1.86 \mathrm{~cm})$. Thus the APA characteristics between conditions were not likely attributable to a changes in the initial position of the CoM.

\section{Experiment 1: Random Perturbations}

In experiment 1 , lateral weight transfer before step initiation was unexpectedly perturbed by randomly introducing a lateral frictional resistance in $25 \%$ of the trials. The summary data depicting the effects observed with the postural perturbation are presented in Fig. 2. Because the perturbation was unexpected by the subjects, it had no influence on 
the initial onset time of the APAs \{i.e., the onset of the ML [Friedman $\mathrm{Chi}^{2}{ }_{(n=11, \mathrm{dl}=2)}=0.73 ; P<0.70$ ] or $\mathrm{AP}$ [Friedman $\mathrm{Chi}^{2}{ }_{(n=11, \mathrm{dl}=2)}=1.28 ; P<0.53$ ] displacement of the $\mathrm{CoP}\}$. On average, subjects started to transfer their weight $208 \mathrm{~ms}( \pm 37)$ after the light cue. However, the unexpected perturbation provided resistance to the ML APA by adding a lateral frictional resistive force at the pelvis approximately equal to $10 \%$ of subjects' body weight $(55.3 \pm 11.3 \mathrm{~N}$ or $9.7 \% \pm 1.5 \%$ body wt). As a result, the mean ML APA duration increased over unperturbed trials by $140 \mathrm{~ms}$ [Friedman $\mathrm{Chi}_{(n=11, \mathrm{dl}=2)}^{2}=18.73 ; P<0.001$; Fig. $\left.3 A\right]$ while the

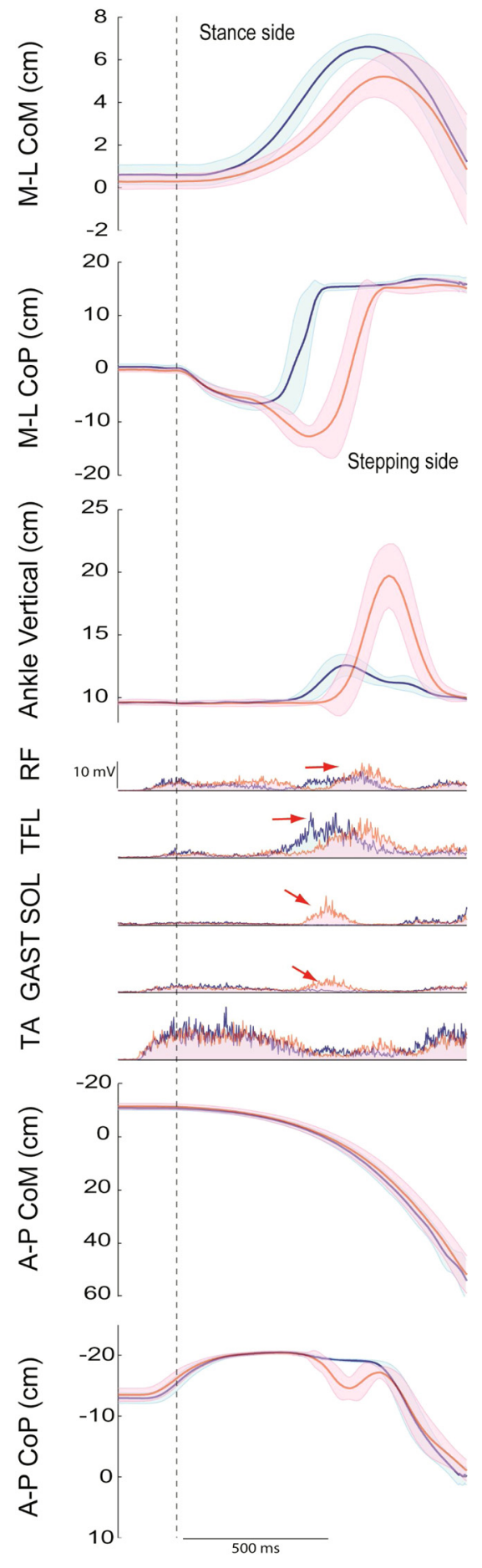

amplitude increased by $37 \%$ [Friedman $\mathrm{Chi}^{2}{ }_{(n=11, \mathrm{dl}=2)}=$ 13.273; $P<0.002$; Fig. 3B]. Despite the modulatory increase in APA duration and amplitude, the velocity [Friedman $\left.\mathrm{Chi}^{2}{ }_{(n=11, \mathrm{dl}=2)}=12.181 ; P<0.003\right]$ and displacement amplitude [Friedman $\mathrm{Chi}^{2}{ }_{(n=11, \mathrm{dl}=2)}=16.909 ; P<0.003$ ] of the CoM motion in the ML direction were reduced at the onset of the first step (Fig. 2). The CoM velocity was slower during the ON condition $(14.1 \pm 2.8 \mathrm{~cm} / \mathrm{s})$ compared with either the OFF condition $(18.6 \pm 3.0 \mathrm{~cm} / \mathrm{s} ; P<0.011, z=$ $2.93)$ or the BASE condition $(17.8 \pm 3.4 \mathrm{~cm} / \mathrm{s} ; P<0.023, z=$ $2.66)$, and the displacement was smaller in the ON condition $(4.9 \pm 0.9 \mathrm{~cm})$ compared with OFF $(6.0 \pm 1.0 \mathrm{~cm} ; P<0.011$, $z=2.93)$ and BASE $(5.8 \pm 0.9 \mathrm{~cm} / \mathrm{s} ; P<0.011, z=2.93)$ conditions. Furthermore, the postural perturbation did not modify the AP APA duration [Friedman $\mathrm{Chi}^{2}{ }_{(n=11, \mathrm{dl}=2)}=3.82$; $P<0.15$ ] or amplitude [Friedman $\mathrm{Chi}^{2}{ }_{(n=11, \mathrm{dl}=2)}=0.55$; $P<0.77$ ] prior step onset (Fig. 2). Thus the postural perturbation only influenced the control characteristics of the ML APA.

In the locomotion system, the perturbation affected the step onset timing [Friedman $\mathrm{Chi}_{(n=11, \mathrm{dl}=2)}^{2}=17.64 ; P<0.001$ ], which was delayed by $\sim 100 \mathrm{~ms}$ when the perturbation was ON $(525 \pm 86 \mathrm{~ms})$ compared with the BASE trials $(403 \pm 59 \mathrm{~ms}$; $P<0.011, z=2.93)$ and to the OFF trials $(421 \pm 66 \mathrm{~ms}$; $P<0.011, z=2.92$; Fig. 3C). To confirm that the step onset delay was not just a mechanical effect of the postural perturbation, it was important to verify if the step motor command was also delayed by the perturbation. Although the step motor command likely involved more muscles than were recorded, including deeper and less accessible hip flexor muscles such as iliopsoas, we used the onset of the EMG activity of the rectus femoris, a hip flexor, and the tensor fasciae latae, a hip rotator and abductor, as representative markers for the onset of the step motor command. Nonparametric ANOVA showed that the onset of the initial step limb rectus femoris [Friedman $\mathrm{Chi}^{2}{ }_{(n=11, \mathrm{dl}=2)}=$ 16.91; $P<0.001$ ] changed in response to the perturbation (Fig. 2). Post hoc analysis indicated that the onset of rectus femoris was delayed by $\sim 100 \mathrm{~ms}$ in the ON trials $(557 \pm 92 \mathrm{~ms})$ compared with the BASE trials $(427 \pm 64 \mathrm{~ms} ; P<0.011, z=$ $2.93)$ and to the OFF trials ( $437 \pm 70 \mathrm{~ms} ; P<0.011, z=2.93$; Fig. 3C). Similar results were observed for the onset of the tensor fasciae latae [Friedman $\mathrm{Chi}^{2}{ }_{(n=11, \mathrm{dl}=2)}=13.82 ; P<$ $0.001]$, which was delayed in the ON trials $(489 \pm 87 \mathrm{~ms})$ compared with the BASE trials $(374 \pm 60 \mathrm{~ms} ; P<0.014, z=$

Fig. 2. Averaged time profiles during random postural perturbations. Averaged data from 1 representative subject during step initiation without (average of 10 baseline trials in blue) and with (average of $15 \mathrm{ON}$ trials in red) a postural perturbation. All records were synchronized with the onset of the net CoP displacement (vertical dotted line) at time zero. The shaded regions represent \pm 1 SD. The lateral displacement of the body center of mass (CoM) shows a smaller displacement for the perturbation ON condition. The lateral displacement of the $\mathrm{CoP}$ under the stepping side is increased in amplitude and duration to overcome the resistance applied to the pelvis. The vertical displacement of the stepping ankle marker shows a delay in the 1st step timing and increased step height. The mean EMG activity of stepping leg muscles shows a delay of the step command [illustrated by the rectus femoris (RF) and the tensor fasciae latae (TFL) activity], a modification of the step motor command with the appearance of a phasic activation bursts in soleus (SOL) and gastrocnemius medialis (GAST) when a postural perturbation is randomly applied, whereas no modification in the onset of the tiabialis anterior (TA) activity was observed. The anteroposterior (AP) displacement of the CoM and the CoP were unmodified for the perturbation $\mathrm{ON}$ condition. 

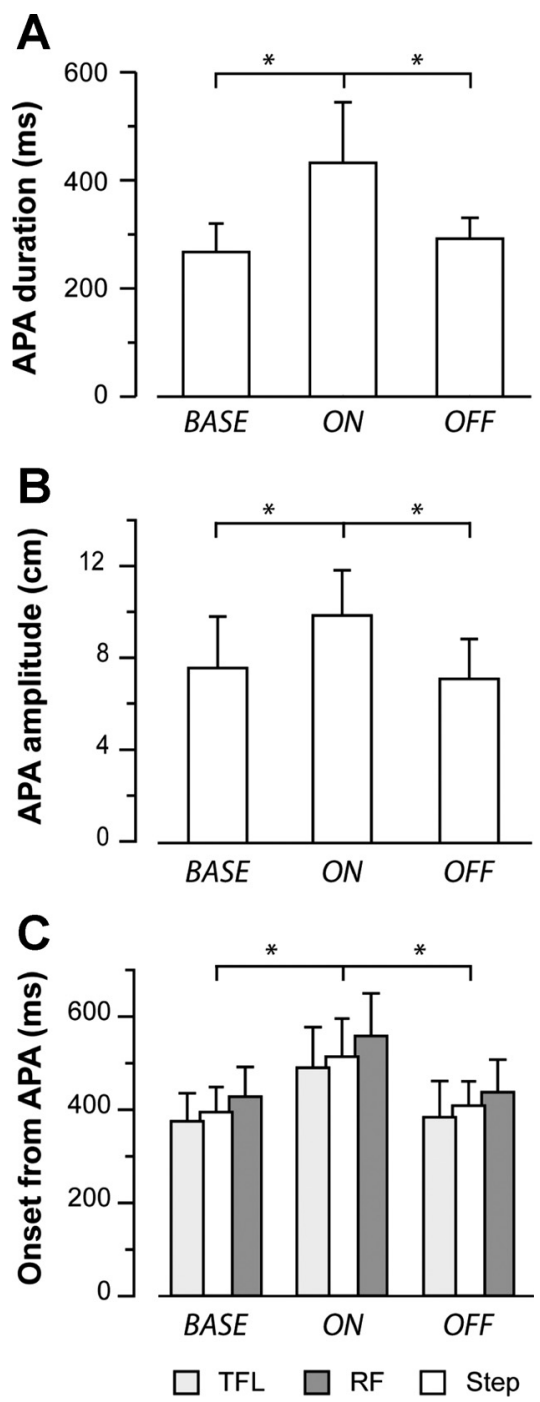

Fig. 3. Effects of a random postural perturbation. Group averages ( $\pm 1 \mathrm{SD})$ of the duration $(A)$ and the amplitude $(B)$ of the ML anticipatory postural adjustments (APAs) and the onset $(C)$ of the TFL (light grey), RF: (dark grey), and the 1 st step (white) relative to the onset of the APA. *Significant differences at $P<0.05$ between the $\mathrm{ON}$ condition and the 2 other conditions, which were not different.

$2.85)$ and to the OFF trials ( $383 \pm 77 \mathrm{~ms} ; P<0.011, z=2.93$; Fig. $3 C$ ). These results show that the step motor command was delayed with the postural perturbation agreeing with the interactive model of posture and locomotion coupling.

In addition to the onset timing delay, some of the characteristics of the first step execution were also modified following the postural perturbation (Fig. 4). The duration of the first step was shorter by $\sim 70 \mathrm{~ms}$ [Fig. $4 A$; Friedman $\mathrm{Chi}^{2}{ }_{(n=11, \mathrm{dl}=2)}=$ 16.55; $P<0.001$; ON: $499 \pm 59 \mathrm{~ms}$; BASE: $570 \mathrm{~ms} \pm 66$; $P<0.011, z=2.93$; OFF: $563 \pm 54 \mathrm{~ms} ; P<0.011, z=2.93]$ and the foot landed more laterally resulting in a wider base of support at first step touchdown [Fig. $4 C$; Friedman $\mathrm{Chi}^{2}{ }_{(n=11, \mathrm{dl}=2)}=$ 17.07; $P<0.001$; ON: $-0.1 \pm 3.4 \mathrm{~cm}$; BASE: $-5.8 \pm 3.5$ $\mathrm{cm} ; P<0.011, z=2.93$; OFF: $6.0 \pm 3.7 \mathrm{~cm} ; P<0.011, z=$ 2.93]. The foot elevation was also increased by $35 \%$ [Fig. $4 B$; Friedman $\mathrm{Chi}^{2}{ }_{(n=11, \mathrm{dl}=2)}=16.91 ; P<0.001 ; \mathrm{ON}: 19.1 \pm$ $1.7 \mathrm{~cm}$; BASE: $14.3 \pm 1.7 \mathrm{~cm} ; P<0.011, z=2.93$; OFF: 14.5 $\mathrm{cm} \pm 1.4 ; P<0.011, z=2.93]$. This increase in height of the step was associated with a unique activation of the ankle plantar flexor muscles (soleus and gastrocnemius medialis) that occurred more often when the perturbation was ON (Fig. 2). Although activity of the gastrocnemius medialis was sometimes observed when there was no perturbation (BASE: $20.0 \pm$ 26.5\%; OFF: $23.0 \pm 29.8 \%$ ), the frequency of trials with a gastrocnemius medialis burst around step onset increased markedly during perturbation trials [Friedman $\mathrm{Chi}^{2}{ }_{(n=11, \mathrm{dl}=2)}=17.63 ; P<$ 0.001; ON: $89.8 \pm 15.6 \% ; P<0.011, z=2.93$ ]. However,
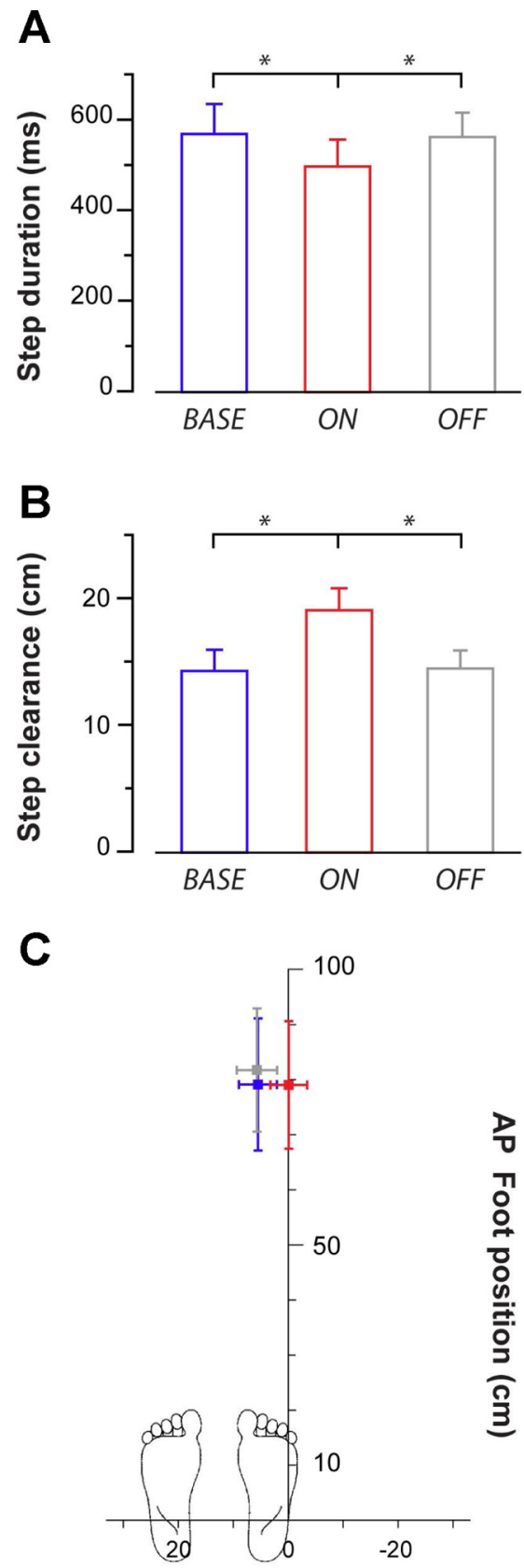

\section{Foot position (cm)}

Fig. 4. First step characteristics changes following the random postural perturbations. Group averages $( \pm 1 \mathrm{SD})$ of the step duration $(A)$, step clearance $(B)$, and length and width $(C)$. The step was shorter in time, higher and placed more laterally, but did not change in length. *Significant differences at $P<$ 0.05 between the ON condition (red) and the two other condition (BASE: blue; OFF: grey), which were not different. 
increased hip flexor activity may also have contributed to the increase in step height in the ON condition compared with BASE trials. Collectively, these results indicate immediate modifications of the step motor command due to the postural perturbation that allowed the subjects to maintain the same step length (Fig. $4 C$ ) with the perturbation (ON: $79.1 \pm 11.6 \mathrm{~cm})$ as without the perturbation (BASE: $79.1 \pm 12.0 \mathrm{~cm}, P<1.0, z=$ 0.56; OFF: $81.7 \pm 11.2 \mathrm{~cm} ; P<0.13, z=2.04)$.

\section{Experiment 2: Systematic Perturbations}

Within a week following experiment 1 , subjects underwent experiment 2. After 10 preadaptation trials (PRE) without perturbation, subjects performed 100 trials with the perturbation (ADAPT). Similar to the random perturbation trials (ON condition) in experiment 1, immediate modifications were found for both APA and locomotion parameters (Fig. 5). In the first block of 10 trials with systematic perturbations (A1), the APA duration $(435 \pm 92 \mathrm{~ms})$ and amplitude $(10.8 \pm 1.8 \mathrm{~cm})$ became larger compared with the preadaptation trials $(271 \pm$ $61 \mathrm{~ms}, P<0.014$ and $7.0 \pm 2.1 \mathrm{~cm}, P<0.014$, respectively). The onset of the stepping movement during A1 was delayed (PRE: $421 \pm 79 \mathrm{~ms} ;$ A1: $524 \pm 69 \mathrm{~ms} ; P<0.014$ ) along with the step motor command (e.g., rectus femoris onset: PRE: $438 \pm 84$ ms; A1: $547 \pm 79 \mathrm{~ms} ; P<0.014$; and tensor fasciae latae onset: PRE: $383 \pm 84 \mathrm{~ms}$; A1: $476 \pm 91 \mathrm{~ms} ; P<0.014)$, the step duration decreased (PRE: $561 \pm 55 \mathrm{~ms}$; A1: $492 \pm 37 \mathrm{~ms}$; $P<0.018$ ) while the step clearance was increased (PRE: $13.9 \pm 1.4$ $\mathrm{cm}$; A1: $17.8 \pm 1.6 \mathrm{~cm} ; P<0.014)$. The frequency of trials with a gastrocnemius medialis burst associated with step onset also increased during the first block with the systematic perturbation (PRE: $27.3 \pm 39.3 \%$; A1: 88.2 $\pm 21.8 \% ; P<0.031$ ). In contrast with experiment 1 , first step width was not statistically different $(P<0.132)$ during the A1 trials $(3.0 \pm 3.4$ $\mathrm{cm})$ from PRE adaptation trials $(4.8 \pm 3.2 \mathrm{~cm})$ indicating subjects readapted their ML foot placement within 10 consecutive perturbed trials. Furthermore, the CoM lateral displacement amplitude and duration were not significantly modified after 10 perturbation trials (A1: $5.8 \pm 0.7 \mathrm{~cm}$ and $602 \pm 74 \mathrm{~ms}$, respectively) from the PRE adaptation condition (PRE: $5.8 \pm$ $0.8 \mathrm{~cm}, P<0.59$ and $578 \pm 69 \mathrm{~ms}, P<0.36$, respectively) showing again that the readaptation of lateral weight transfer was achieved within 10 consecutive perturbed trials.

Immediately following the completion of the 100 adaptation trials, the perturbation was unexpectedly removed and subjects performed 10 postadaptation trials (POST). Across the ten POST trials, the duration $(301 \pm 31 \mathrm{~ms})$ and the amplitude $(6.3 \pm 1.0 \mathrm{~cm})$ of the APAs returned to their PRE adaptation values $(P<0.619$ and $P<0.853$, respectively). Similar findings were observed for step onset time (POST: $446 \pm 48$ ms; $P<0.619$ ), step motor command (e.g., rectus femoris POST: $482 \pm 55 \mathrm{~ms} ; P<0.202$; tensor fasciae latae POST: $421 \pm 51 \mathrm{~ms} ; P<0.202$; Fig. 5), step duration (POST: $554 \pm$ $52 \mathrm{~ms} ; P<1.00$ ), as well as for ML displacement of the CoM (POST: $5.9 \pm 0.9 \mathrm{~cm} ; P<1.00$; Fig. $6 A$ ) and step width (POST: $1.6 \pm 0.1 \mathrm{~cm} ; P<0.438$; Fig. $6 B$ ), which were all on average statistically not different from their PRE adaptation values. On the other hand, step clearance remained higher (POST: $15.6 \pm 2.3 \mathrm{~cm} ; P<0.018$ ) compared with PRE adaptation values. However, the frequency of trials with a gastrocnemius medialis burst associated with step onset was not different from preadaption values $(P<0.101 ;$ Fig. $6 C)$. All these changes were observed while subjects maintained the same step length for all trials [Friedman $\mathrm{Chi}^{2}{ }_{(n=11, \mathrm{dl}=11)}=$ 9.92; $P<0.538]$.

To assess possible aftereffects lasting less than the 10 POST trials, we compared the preadaptation trials to the first postadaptation trial (T1) and each consecutive block of three trials (T2-4; T5-7; T8-10). Although it did not reach statistical significance after Bonferroni correction, the APA of the first postadaptation trial tended to be longer (T1: $271 \pm 60 \mathrm{~ms} ; P<$ $0.082)$ and larger (T1: $7.0 \pm 2.1 \mathrm{~cm} ; P<0.124)$. This trend of an increase in the APA amplitude and duration was not sufficient to downscale the CoM motion in the ML direction, which was still longer (T1: $749 \pm 135 \mathrm{~ms} ; P<0.007$; Fig. 5) and larger (T1: $9.5 \pm 2.3 \mathrm{~cm} ; P<0.007$; Fig. $6 A$ ) for the first POST trial compared with PRE values. This explained the landing position of the foot, which was more medial during the first POST trial (T1: $17.7 \pm 7.6 \mathrm{~cm} ; P<0.007)$ than during preadaptation (Fig. $6 B$ ) in compensation for the increased CoM body motion towards the single support limb side. It is noteworthy that the readjustment to PRE trial values for step onset
Fig. 5. Effects of a systematic postural perturbation on the timing of the events. Group averages ( $\pm 1 \mathrm{SD}$ ) of the timing of postural and locomotor's events relative to the onset of the anticipatory postural adjustments are presented for experiment 2 . *Significant difference at $P<0.05$ between PRE adaptation trials and the 1st block of adaptation (A1). §Significant difference at $P<0.05$ between PRE adaptation and the 1st POST adaptation trial (T1).

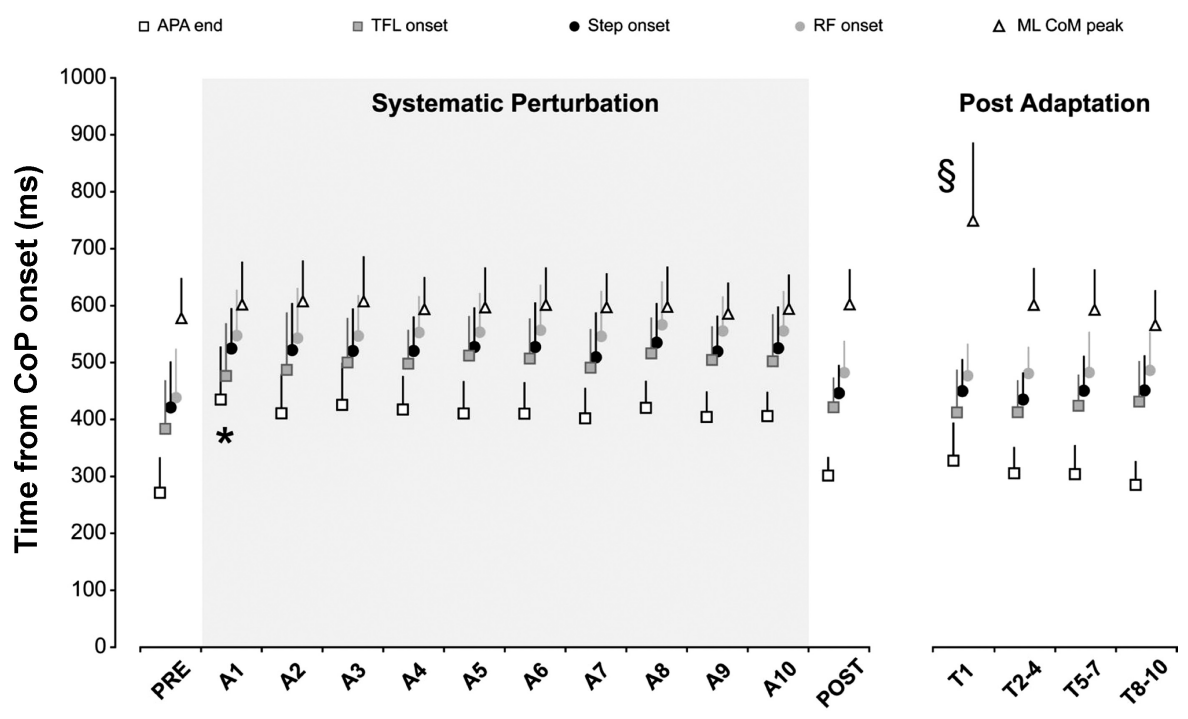



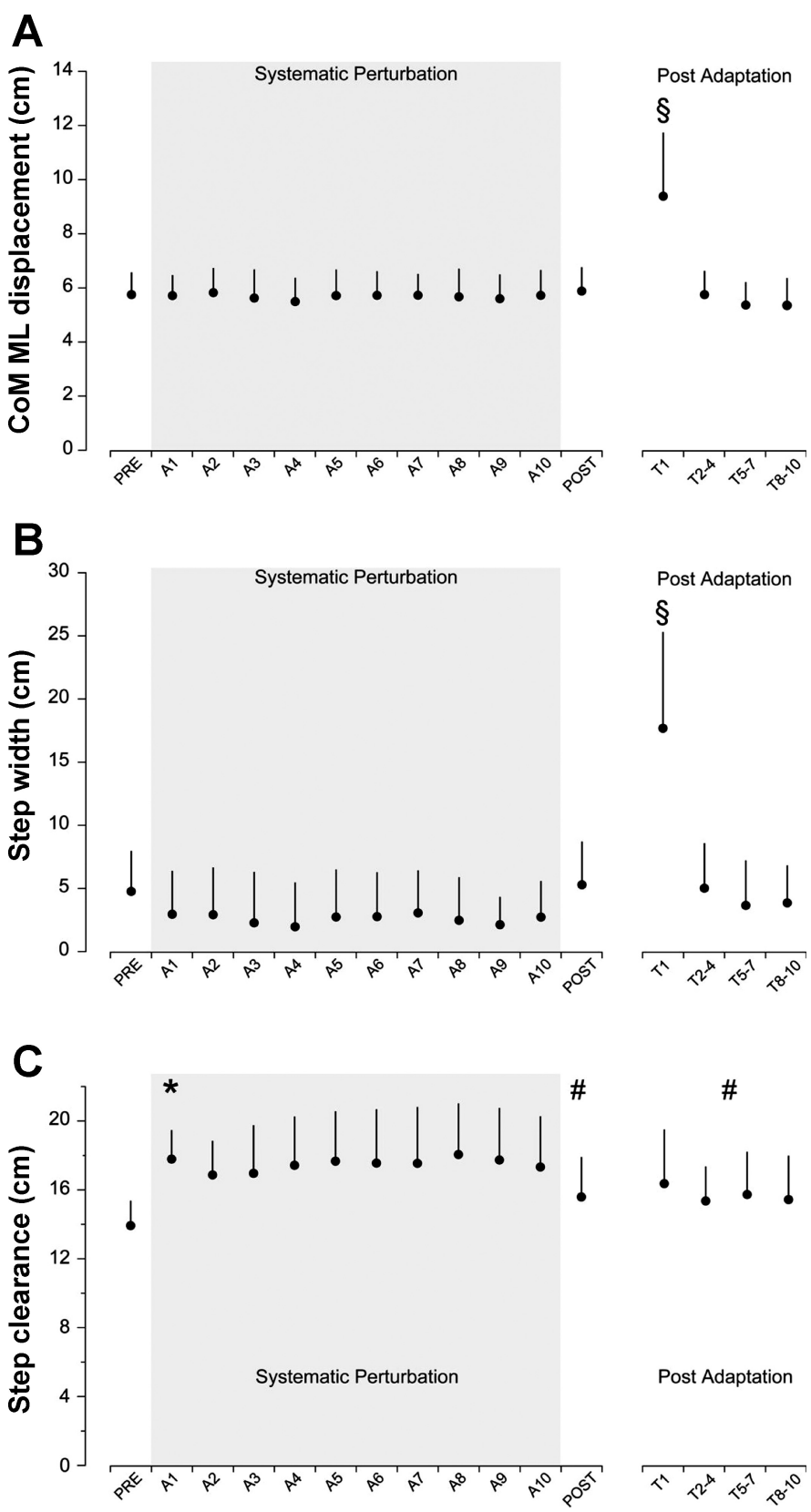

Fig. 6. Effects of a systematic postural perturbation. Group averages ( $\pm 1 \mathrm{SD})$ for ML displacement of the CoM $(A)$, step width $(B)$, and step clearance $(C)$ are presented for experiment 2. *Significant difference at $P<0.05$ between PRE adaptation trials and the 1st block of adaptation (A1). \#Significant difference at $P<0.05$ between PRE and POST adaptation trials. \$Significant difference at $P<0.05$ between PRE adaptation and the 1st POST adaptation trial (T1).

(T1: $449 \pm 79 \mathrm{~ms} ; P<0.523$ ), step motor command (T1 rectus femoris: $476 \pm 55 \mathrm{~ms} ; P<0.365$; tensor fasciae latae: $412 \pm$ $74 \mathrm{~ms} ; P<0.558$ ), and step duration (T1: $538 \pm 59 \mathrm{~ms} ; P<$ 0.991 ) occurred immediately during the first POST trial (Fig. 5). In contrast, step clearance showed no tendency to a return to the PRE adaptation values since all trials of postadaptation were higher $(P<0.04$; Fig. $6 C)$.

\section{DISCUSSION}

We showed that following unexpected perturbations disrupting ML APAs before voluntary stepping, postural parameters were rapidly modified together with a delay in the initiation timing and modification of other locomotion parameters. These results supported the hypothesis of an interactive mode of posture and locomotion coupling whereby the release of locomotion is withheld until the APAs reach certain state conditions likely reflecting an estimation of whole body stability. Furthermore, first step execution (duration, clearance, and width) was adapted together with the postural changes suggesting that a prediction of the consequences of the APAs might have been used to modify the step command by updating the putative internal model for posture and locomotion coupling. When systematic perturbations were applied and then suddenly withheld, aftereffects were mainly observed only in the first trial. Only a change in step clearance was sustained during all postadaptation trials. This immediate retention of some features of the adapted pattern indicated the presence of a predictive feedforward mechanism contributing to posture and locomotion coupling, whereas the more acute and transient modifications indicated rapid online modification of posture and locomotion.

\section{Posture and Locomotion Coupling}

Two modes of coordination between posture and movement have been proposed (Massion 1992, 1994; Schepens and Drew 2003; Schepens et al. 2008) leading to opposite predictions for unexpected disruption of the postural transition from bipedal to single limb support. Normally, a unipedal stance condition is achieved before stepping. Hence, in unexpected perturbation trials, online modification of the ML APA characteristics (e.g., duration and amplitude) was needed to achieve comparable postural state conditions for the CoM position and motion relative to the changing base of support as occurs during unperturbed stepping (Mille et al. 2007, 2009; Mouchnino et al. 2012). Despite the adaptive changes in APA parameters, the CoM motion was reduced during these trials compared with unperturbed trials. Because subjects did not know whether a perturbation would occur until they initiated the APAs, their onset timing was not different from the unperturbed trials. Thus the delay in step onset timing indicated that the motor command for stepping was suppressed, apparently until the estimated conditions for stability were achieved. These results are consistent with the interactive model of posture and locomotion coordination (Fig. 1). Alternatively, in the independent sequential model, an unexpected postural perturbation should not affect the step onset timing. As this outcome was rejected, we provide evidence for the interdependence of locomotor control with the predicted or prevailing postural state conditions. The fact that step onset occurred earlier when a postureassisting protocol was previously applied (i.e., when body weight transfer was mechanically assisted so that the postural state conditions were achieved more quickly) is in agreement with this interactive model (Mille et al. 2007). This mode of coordination implies a hierarchy in posture and locomotion coupling where postural events prevail upon locomotor execution.

\section{Online Locomotor Adaptations}

Historically, APAs were thought to involve an efferent copy of the motor command for an intended action that acts on the postural networks (Massion 1992, 1994). This explains why 
APAs are adjusted to the characteristics of the planned movement (Lee et al. 1987; Rogers and Pai 1990; Lepers and Brenière 1995). The fact that step onset time was delayed with the random postural perturbations indicated that the opposite case is also true whereby the CNS estimated the potential consequences to stability of the impending action, a process that involves an internal model of one's own body and the external world (Wolpert et al. 1995; Kawato 1999). In effect, these changes in motor prediction offset the anticipated disruptions to posture caused by stepping while minimizing the inherent delays in feedback-based postural corrections (Miall and Wolpert 1996).

Some of the adaptation effects were consistent with a role for motor prediction in posture and locomotion coupling. First, compared with unperturbed trials, step width became more lateral for unexpected perturbations and more medial when expected perturbations were suddenly terminated following practice. Normally, in step initiation, frontal plane body motion appears to be controlled ballistically (Lyon and Day 1997), which implies that the CNS scales in advance the direction and magnitude of the initial lateral "throw" of the body in correspondence with the intended location of ML foot placement. By altering the prestep postural movement, the ML foot position was consequently adapted to ensure stability at step landing. This likely explains why after 100 adaptation trials, step width was rapidly recorrected after the first POST trial to avoid falling during unperturbed stepping. Second, with the random perturbations, step height and phasic ankle extensor muscle activity increased. Moreover, these adaptations in locomotion were not due to changes in AP APAs for forward propulsion, which could have more directly altered stepping performance. Instead, disruption of frontal plane postural control precipitated locomotor adjustments to maintain stability and the efficiency of the first step (e.g., maintaining step length).

\section{Longer Term Adaptation of Posture-Locomotion Coupling}

Our results indicated that the spatial and temporal parameters of posture and locomotion coupling were not adapted similarly, as previously observed for split-belt treadmill walking (Malone and Bastian 2010; Torres-Oviedo and Bastian 2010). Whereas the step height adjustment showed a robust and persistent adaptation aftereffect, the aftereffects for lateral CoM motion and ML foot placement were more quickly readapted to PRE values. These modifications are again consistent with a predictive feedforward adaptation process (Mille et al. 2012). We expected that repetitive postural perturbations altering APA characteristics would show aftereffect changes in step onset timing. Although there was a trend for step onset EMG activity to be later than during preadaptation, this difference was not statistically significant. Hence, the temporal sequencing between the instant of ML APA onset and the time of step initiation may be indicative of a feedback-mediated adaptation reflecting the postural state conditions used to trigger the step. The fact that a previous reversal of the experimental paradigm (i.e., by assisting the ML APA) showed complimentary results, more rapid step onset timing with random ML APA assistance (Mille et al. 2007), and no timing adaptation after systematic assistance (Mille et al. 2009) further supports this hypothesis.
CNS Regions Involved in Adapting Posture and Locomotion coupling

Adaptive locomotion through variable and unpredictable environmental terrain requires continuous modification of walking parameters with complimentary recalibration of postural orientation and stabilization. Although the spinal cord, brain stem, basal ganglia, and motor cortex contribute importantly to the control of posture and locomotion, the cerebellum plays a prominent role in motor adaptation (Bastian 2008). Involvement of the cerebellum in adaptively modifying volitional and automatic processes for posture and locomotion control likely occurs through interaction with cortical and brainstem regions. For example, sensory feedback mediated by the spinocerebellar tract and feedforward information from the cerebral cortex over the olivocerebellar tract may be important in these functions (Takakusaki 2013). Furthermore, the cerebellum is especially important in mediating predictive feedforward adaptation of posture and locomotion such as the observed aftereffect changes in the first step width and height and CoM motion (Morton and Bastian 2006; Choi and Bastian 2007). In contrast, cerebellar involvement in reactive feedback adaptations is less likely since people with cerebellar damage can make normal rapid adaptations in locomotion (Morton and Bastian 2006). Thus the rapid adaptive changes that occurred during the random perturbation trials and early in the practice phase, but not in the postadaptation phase of the systematic perturbations, were consistent with the presence of reactive feedback-driven adaptations. Previous findings have suggested that spinal mechanisms may be important for such reactive adaptations (Rossignol et al. 2006). In particular, sensory afferent information from receptors in the lower limbs signaling limb loading conditions and hip postural orientation implicated in stance to swing phase gait transitions are relevant to the reactive adaptations that we observed (Pearson 2004). The current findings are also relevant for neurological conditions that impair posture and locomotion such as "start-hesitation" delays in gait initiation in Parkinson's disease where the normal coupling between these elements may be disrupted (Rogers et al. 2011).

In summary, our novel postural perturbation method allowed us to probe the coordinative structure and adaptive behavior of posture and locomotion integration during the initiation of walking. We found that the timing release and other operational parameters of locomotion were dependent on the postural constraints that occurred before stepping. Furthermore, during random perturbation trials, first step characteristics were rapidly adapted with the changes the APAs suggesting that a prediction of the evolving state of postural stability might be used to modify the step motor command by reconfiguring the presumed internal model for posture and locomotion coupling. When systematic perturbations were applied and then suddenly removed, aftereffects occurred mainly in the first trial and were immediately readapted to preperturbation levels apparently again to preserve balance stability. Only the change in step clearance was sustained during postadaptation trials possibly as an adaptive strategy to guard against potential foot-placement errors affecting landing stability or to avoid tripping related to pelvic drop affecting foot-ground clearance regulated by the stance side hip abductor/adductor musculature that could vary with APA changes due to the ML perturbation. Overall, our 
results showing both rapid but transient reactive adaptations in the posture and gait assembly and more durable practicedependent changes suggesting feedforward adaptation indicated newly identified features of postural and locomotion coordination that extend prior observations of motor adaption during ongoing locomotion to the transition from standing still to walking. Finally, the findings have implications for current approaches to neurorehabilitation that separately target postural training or gait training in Parkinson's disease and other conditions, which may benefit from shifting attention to improving the adaptive coupling between posture and locomotion interactions rather than their isolated functions.

\section{GRANTS} 16780 .

This work was supported by National Institute of Aging Grant RO1-AG-

\section{DISCLOSURES}

No conflicts of interest, financial or otherwise, are declared by the author(s).

\section{AUTHOR CONTRIBUTIONS}

Author contributions: M.-L.M., M.S., and M.W.R. conception and design of research; M.-L.M., M.S., and M.W.R. performed experiments; M.-L.M. and M.S. analyzed data; M.-L.M., M.S., and M.W.R. interpreted results of experiments; M.-L.M. prepared figures; M.-L.M. and M.W.R. drafted manuscript; M.-L.M. and M.W.R. edited and revised manuscript; M.-L.M., M.S., and M.W.R. approved final version of manuscript.

\section{REFERENCES}

Bastian AJ. Understanding sensorimotor adaptation and learning for rehabilitation. Curr Opin Neurol 21: 628-633, 2008.

van Boxtel GJ, Geraats LH, van den Berg-Lenssen MM, Brunia CH. Detection of EMG onset in ERP research. Psychophysiology 30: 405, 1993.

Brenière $\mathbf{Y}$, Do $\mathbf{M C}$, Bouisset $\mathbf{S}$. Are dynamic phenomena before stepping essential to walking? J Mot Behav 19: 62-76, 1987.

Brunt D, Lafferty MJ, Mckeon A, Goode B, Mulhausen C, Polk P. Invariant characteristics of gait initiation. Am J Phys Med Rehabil 70: 206-212, 1991.

Choi JT, Bastian AJ. Adaptation reveals independent control networks for human walking. Nat Neurosci 10: 1055-1062, 2007.

Crenna P, Frigo C. A motor programme for the initiation of forward-oriented movements in humans. J Physiol 437: 635-653, 1991.

Kawato M. Internal models for motor control and trajectory planning. Curr Opin Neurobiol 9: 718-727, 1999.

Lee WA, Buchanan TS, Rogers MW. Effects of arm acceleration and behavioral conditions on the organization of postural adjustments during arm flexion. Exp Brain Res 66: 257-270, 1987.

Lepers R, Brenière $\mathbf{Y}$. The role of anticipatory postural adjustments and gravity in gait initiation. Exp Brain Res 107: 118-124, 1995.

Lidierth M. A computer based method for automated measurement of the periods of muscular activity from an EMG and its application to locomotor EMGs. Electroencephalogr Clin Neurophysiol 64: 378-380, 1986.

Lyon IN, Day BL. Control of frontal plane body motion in human stepping. Exp Brain Res 115: 345-356, 1997.
Malone LA, Bastian AJ. Thinking about walking: effects of conscious correction vs. distraction on locomotor adaptation. J Neurophysiol 103: 1954-1962, 2010.

Mann RA, Hagy JL, White V, Liddell D. The initiation of gait. J Bone Joint Surg Am 61: 232-239, 1979.

Massion J. Movement, posture and equilibrium: interaction and coordination. Prog Neurobiol 38: 35-56, 1992.

Massion J. Postural control system. Curr Opin Neurobiol 4: 877-887, 1994.

Mille ML, Creath RA, Prettyman MG, Johnson Hilliard M, Martinez KM, Mackinnon CD, Rogers MW. Posture and locomotion coupling: a target for rehabilitation interventions in persons with Parkinson's disease. Parkinsons Dis 2012: 754186, 2012.

Mille ML, Hilliard MJ, Martinez KM, Simuni T, Zhang Y, Rogers MW. Short-term effects of posture-assisted step training on rapid step initiation in Parkinson's disease. J Neurol Phys Ther 33: 88-95, 2009.

Mille ML, Johnson Hilliard M, Martinez KM, Simuni T, Rogers MW. Acute effects of a lateral postural assist on voluntary step initiation in patients with Parkinson's disease. Mov Disord 22: 20-27, 2007.

Mille ML, Johnson ME, Martinez KM, Rogers MW. Age-dependent differences in lateral balance recovery through protective stepping. Clin Biomech (Bristol, Avon) 20: 607-616, 2005.

Mille ML, Mouchnino L. Are human anticipatory postural adjustments affected by a modification of the initial position of the center of gravity? Neurosci Lett 242: 61-64, 1998.

Mille ML, Rogers MW, Simoneau M. Adaptation of step initiation to postural perturbation In: Program No 566. 12 Neuroscience Meeting Planner. Orlando, FL: Society for Neuroscience, 2002.

Morton SM, Bastian AJ. Cerebellar contributions to locomotor adaptations during splitbelt treadmill walking. J Neurosci 26: 9107-9116, 2006.

Mouchnino L, Robert G, Ruget H, Blouin J, Simoneau M. Online control of anticipated postural adjustments in step initiation: evidence from behavioral and computational approaches. Gait Posture 35: 616-620, 2012.

Pearson KG. Generating the walking gait: role of sensory feedback. Prog Brain Res 143: 123-129, 2004.

Rogers MW, Hedman LD, Johnson ME, Cain TD, Hanke TA. Lateral stability during forward-induced stepping for dynamic balance recovery in young and older adults. J Gerontol A Biol Sci Med Sci 56: M589-M594, 2001.

Rogers MW, Hilliard MJ, Martinez KM, Zhang Y, Simuni T, Mille ML. Perturbations of ground support alter posture and locomotion coupling during step initiation in Parkinson's disease. Exp Brain Res 208: 557-567, 2011.

Rogers MW, Pai YC. Dynamic transitions in stance support accompanying leg flexion movements in man. Exp Brain Res 81: 398-402, 1990.

Rogers MW, Pai YC. Patterns of muscle activation accompanying transitions in stance during rapid leg flexion. J Electromyogr Kinesiol 3: 149-156, 1993.

Rossignol S, Dubuc R, Gossard JP. Dynamic sensorimotor interactions in locomotion. Physiol Rev 86: 89-154, 2006.

Scarchilli K, Vercher JL. The oculomanual coordination control center takes into account the mechanical properties of the arm. Exp Brain Res 124: 42-52, 1999.

Schepens B, Drew T. Strategies for the integration of posture and movement during reaching in the cat. J Neurophysiol 90: 3066-3086, 2003.

Schepens B, Stapley P, Drew T. Neurons in the pontomedullary reticular formation signal posture and movement both as an integrated behavior and independently. J Neurophysiol 100: 2235-2253, 2008.

Takakusaki K. Neurophysiology of gait: from the spinal cord to the frontal lobe. Mov Disord 28: 1483-1491, 2013.

Torres-Oviedo G, Bastian AJ. Seeing is believing: effects of visual contextual cues on learning and transfer of locomotor adaptation. J Neurosci 30: 17015-17022, 2010.

Wolpert DM, Ghahramani Z, Jordan MI. An internal model for sensorimotor integration. Science 269: 1880-1882, 1995. 